\section{Renal Carcinoma Presenting with Peripheral Arterial Embolisation}

\section{G. J. C. M. NIESSEN and H. VAN URK, Department of \\ Surgery, University Hospital, Rotterdam, The Netherlands}

\section{Case Report}

A 69-year-old man presented with the sudden development of a pale, painful and pulseless left lower leg. Physical examination revealed a varicocele on the left side which had apparently been present for only a few months. There was no history of intermittent claudication. Chest X-ray showed multiple lesions, suggesting metastases in both lungs. Embolectomy under local anaesthesia was performed immediately. Microscopic examination of the thrombus showed tumour cells. Subsequent analysis showed renal function to be abnormal and an intravenous urogram showed a picture suggestive of left renal carcinoma. Surgical resection was not attempted. The patient's general condition deteriorated rapidly and he died 23 days after admission. Autopsy demonstrated a large renal carcinoma with pulmonary metastases and invasive growth in the renal vessels, inferior vena cava and left ureter.

\section{Comment}

Malignant tumour emboli to peripheral arteries are relatively rare and most of them originate from lung tumours (Starr et al., 1981). Until now only one renal carcinoma presenting with peripheral arterial occlusion has been described (Stanley and Eto, 1978). Renal carcinomas are known to present with protean manifestations (Chisholm, 1974); the present case stresses the diversity of symptoms of the disease. The sudden occurrence of peripheral emboli without a history of claudication or cardiac arrhythmia should raise the suspicion of malignancy; further analysis may be decisive for subsequent treatment. However, this diagnostic clue is probably of no prognostic value for the patient as it concerns a late symptom of disseminated disease. It is important to undertake microscopic examination of thrombus material removed after embolectomy.

\section{References}

Chisholm, G. D. (1974). Malignant disease of the kidney. An appraisal of diagnosis and management. Annals of the Royal College of Surgeons of England, 55, 21-30.

Stanley, P. and Eto, R. T. (1978). Arterial embolization of malignant tumor: report of two cases with angiographic findings. Radiology, 126, 93-94.
Starr, D. S., Lawrie, G. M. and Morris, G. C. (1981). Unusual presentation of bronchogenic carcinoma: case report and review of the literature. Cancer, 47, 398-401.

Requests for reprints to: Dr. G. J. C. M. Niessen, Department of Surgery, St. Lambertus Hospital, P.O. Box 98, 5700 AB Helmond, The Netherlands.

\section{Carcinoma of the Seminal Vesicle}

J. J. LANGHAM-BROWN and G. F. ABERCROMBIE, Department of Urology, St Mary's Hospital, Portsmouth

\section{Case Report}

A 21-year-old man was admitted with acute retention of urine due to blood clots. He had had three episodes of haematuria and haematospermia in the preceding 8 weeks. Nine months previously he had noticed an episode of urethral discharge.

Intravenous urography was unremarkable. Urine cultures, including examination for acid fast bacilli, were normal. Blood count and screening tests for venereal disease were also normal. At cystoscopy the bladder was normal. A mass above and to the left of the prostate was felt on bimanual examination. A diagnosis of inflammatory disease was made and the patient discharged on antibiotics.

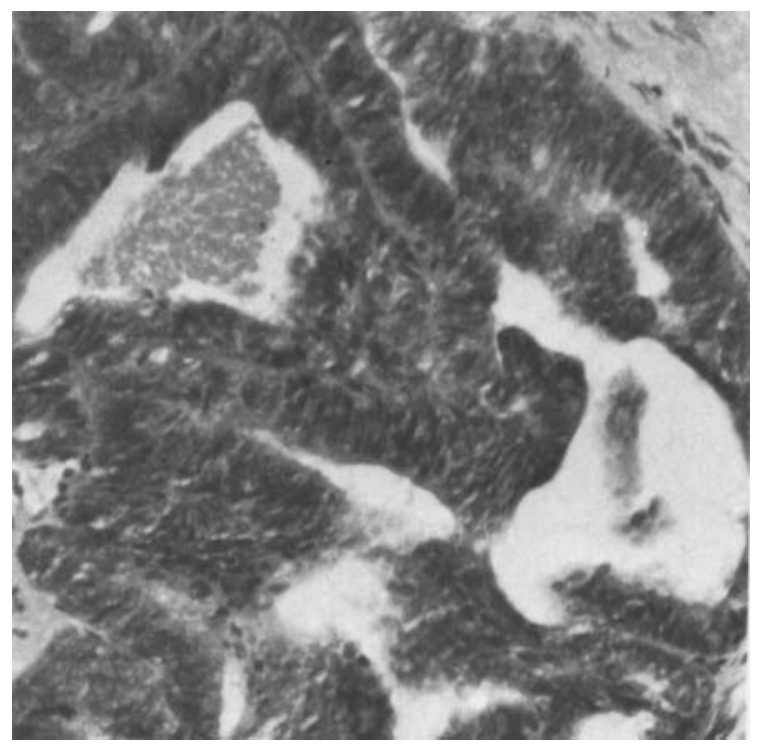

Fig. Trucut biopsy showing tall columnar cell carcinoma of seminal vesicle with poorly defined intraluminal papillae. (H and $\mathrm{E} \times 200$ ). 